\title{
Evaluation of Newer Insecticides for the Management of Brinjal Shoot and Fruit Borer Leucinodes orbonalis (Guenee) (Lepidoptera: Crambidae)
}

\author{
M. Shridhara ${ }^{1 *}$, S.G. Hanchinal ${ }^{1}$, A.G. Sreenivas ${ }^{1}$, A.C. Hosamani ${ }^{1}$ and J.M. Nidagundi ${ }^{2}$ \\ ${ }^{1}$ Department of Agricultural Entomology, ${ }^{2}$ Department of Genetics and Plant Breeding, UAS, \\ Raichur- 584 104, Karnataka, India \\ *Corresponding author
}

\section{A B S T R A C T}

\section{Keywords}

Brinjal shoot and fruit borer,

Leucinodes orbonalis (Guenee), brinjal, new insecticides

\section{Article Info}

Accepted: 26 February 2019 Available Online: 10 March 2019
To evaluate the efficacy of new insecticides against brinjal shoot and fruit borer (BSFB), $L$. orbonalis, field experiment was carried out in a randomized block design with seven treatments (six insecticides and one control) and three replications during kharif 2017 at Main Agricultural Research Station (MARS), Raichur. Among the treatments the lowest mean per cent shoot damage was recorded in the treatment with emamectin benzoate 5 SG (5.41) followed by chlorantraniliprole 18.5 SC (5.83) and flubendiamide 39.5 SC (5.93) and were superior than the untreated control (14.66) after first and second spray. After third spray the lowest mean per cent fruit damage was recorded in the treatment with chlorantraniliprole 18.5 SC (14.32) followed by flubendiamide 39.5 SC (15.78) and emamectin benzoate $5 \mathrm{SG}$ (16.30) and were superior than the other treatments. The highest yield was observed in the treatment with emamectin benzoate $5 \mathrm{SG}\left(25.9 \mathrm{t}^{\mathrm{t}} \mathrm{ha}^{-1}\right)$ followed by flubendiamide $39.5 \mathrm{SC}\left(23.7 \mathrm{t}^{-h^{-1}}\right)$ and chlorantraniliprole $18.5 \mathrm{SC}\left(21.8 \mathrm{t}^{-h^{-1}}\right)$ compared to the untreated control $\left(10.4\right.$ t.ha $\left.^{-1}\right)$ and recorded maximum benefit cost ratio in the field treated with emamectin benzoate 5 SG $(1: 3.17)$ followed by flubendiamide 39.5 SC (1: 2.96) and chlorantraniliprole 18.5 SC (1:2.63).

\section{Introduction}

Brinjal (Solanum melongena L.) is one of the most widely grown solanaceous vegetable and is being grown throughout the year under irrigated condition. A poor man's crop it might be, but brinjal is also called as the King of Vegetables. Due to its nutritive value, consisting of minerals like iron, phosphorus, calcium, folate, and vitamins like A, B and C, unripe fruits are used primarily as vegetable in the country. It is also used as a raw material in pickle making and as an excellent remedy for curing the diabetes. It is also used as a good apetizer. It is a good aphrodisiac, cardiotonic, laxative and reliever of inflammation (Singh et al., 1962). In India brinjal is grown nearly 7.11 lakh ha with a production of 13,558 million tonnes and an average productivity of 19.12 tonnes/ ha (Anon., 2016), making the country the second largest producer after China with a 25 per 
cent of world production share. In Karnataka, brinjal is being grown in an area of 1.58 lakh ha with a production of 402.5 metric tonnes (3.13\% share) and a productivity of 25.4 metric tonnes per hectare (Anon., 2016). Though brinjal is a summer crop, it is being grown throughout the year under irrigated conditions. Hence, it is subjected to attack by number of insect pests right from the nursery stage to till harvesting (Raghupathy et al., 1997). Brinjal is attacked by more than 70 insect pests, among the insect pest infesting brinjal, the major ones are shoot and fruit borer, Leucinodes orbonalis (Guen.), whitefly, Bemisia tabaci (Genn.), leaf hopper, Amrasca biguttula biguttula (Ishida), Epilachna beetle, Henosepilachna vigintioctopunctata (Fab.) and non-insect pest, red spider mite, Tetranychus macfurlanei (Baker and Pritchard). Of these, the brinjal shoot and fruit borer, L. orbonalis is considered as the main constraint as it damages the crop throughout the year. It is known to damage shoot and fruit of brinjal in all stages of its growth. The pest is estimated to cause 70 to 92 per cent yield loss (Vevai et al., 1970; Subbaratnam and Butani, 1982; Reddy and Srinivasa, 2004). The brinjal shoot and fruit borer, L. orbonalis is known to damage shoots and fruits in all stages of plant growth. In early stage of the crop growth, larva bores into the shoots resulting in drooping, withering and drying of the affected shoots. During the reproductive stage, tiny larva bores into the flower buds and fruits, the bored holes are invariably plugged with excreta. The infested fruits become unfit for consumption due to loss of quality and lose their market value. It is also reported that there will be reduction in vitamin $\mathrm{C}$ content to an extent of 68 per cent in the infested fruits (Hemi, 1955). Among the various methods of pest management, the use of insecticides forms the first line of defence against the insect pests. Newer insecticide molecules are better alternative to conventional synthetic insecticides in the context of environmentally benign management tactics so also in order to mitigate the adverse effect on the total environment. In many cases, alternate or ecofriendly method of insect management offer adequate level of pest control with less hazards and safe to non-target organisms. With this background, the present study was undertaken to evaluate the bioefficacy of newer insecticides against the brinjal shoot and fruit borer, L. orbonalis.

\section{Materials and Methods}

To evaluate the efficacy of new insecticides against brinjal shoot and fruit borer, L. orbonalis, field experiment was conducted during Kharif 2017 at Main Agricultural Research Station (MARS), Raichur as an irrigated crop. The experiment was laid out in a Randomized Block Design (RBD) with three replications and seven treatments in a 5 $\mathrm{x} 4$ square meter plot with spacing of $90 \times 45$ $\mathrm{cm}$ and the variety used was "Rayadurga". The foliar treatments were given using knapsack compression sprayer. Three foliar applications were given during the course of investigation on the basis of the ETL of the pest. Observations on pest damage were recorded on ten randomly selected plants prior to the treatment and after imposing the treatment. Post treatment observations were recorded on $1,3,7$, and 14 days after spraying. The fruit yield was recorded plot wise as and when the harvesting was done.

The shoot damage by $L$. orbonalis was assessed based on the total number of shoots and affected shoots in a plot on 10 randomly selected plants and the per cent shoot damage was worked out. The fruit damage by $L$. orbonalis was assessed based on the total number of fruits and the number of damaged fruits in 10 randomly selected plants, and the per cent fruit damage was worked out. The yield of brinjal fruits was recorded from each 
plot on weight basis and computed to per hectare. The per cent data recorded for shoot and fruit damage was converted into corresponding angular transformation (Arcsine) if the values ranged from 0 to 100 for statistical analysis.

\section{Results and Discussion}

At first spray, after fourteen days of imposing treatments the lowest per cent shoot damage was recorded in emamectin benzoate $5 \mathrm{SG}$ (6.60). This was followed by chlorantraniliprole $\quad 18.5 \quad \mathrm{SC} \quad$ (6.91), flubendiamide $39.5 \mathrm{SC}$ (7.12) and all the three treatments were on par with each other but significantly superior over rest of the treatments. The mean lowest per cent shoot damage was recorded in emamectin benzoate 5 SG (5.41). This was followed by chlorantraniliprole 18.5 SC (5.83) and flubendiamide $39.5 \mathrm{SC}$ (5.96) and were superior than the untreated control (14.66). The insecticides in the decreasing order of their efficacy were emamectin benzoate $5 \mathrm{SG}$ $>$ chlorantraniliprole $18.5 \mathrm{SC}>$ flubendiamide 39.5 SC > cyantraniliprole $10 \mathrm{OD}>$ bifenthrin $10 \mathrm{EC}>$ spinosad $45 \mathrm{SC}$ (Table 1).

At Second spray, Fourteen days after imposing the treatment, the lowest shoot damage $(8.57 \%)$ was recorded in emamectin benzoate 5 SG and flubendiamide $39.5 \mathrm{SC}$ $(9.39 \%)$, these treatments were on par with each other and there was no significant difference between these treatments. These were followed by chlorantraniliprole $18.5 \mathrm{SC}$ $(9.71 \%)$ and cyantraniliprole 10 OD (10.57 $\%$ ). Highest shoot damage of 22.79 per cent was observed in untreated control. The mean lowest per cent shoot damage was recorded in emamectin benzoate 5 SG (6.32). This was followed by flubendiamide $39.5 \mathrm{SC}$ (6.94) and chlorantraniliprole 18.5 SC (7.19) and were superior than the untreated control (17.88). The insecticides in the decreasing order of their efficacy were emamectin benzoate $5 \mathrm{SG}>$ flubendiamide $39.5 \mathrm{SC}>$ chlorantraniliprole $18.5 \mathrm{SC}>$ cyantraniliprole $10 \mathrm{OD}>$ bifenthrin $10 \mathrm{EC}>\operatorname{spinosad} 45 \mathrm{SC}$ (Table 2).

At third spray, Fourteen days after imposing the treatment, the lowest fruit damage (16.52\%) was recorded in chlorantraniliprole 18.5 SC, which was significantly superior over rest of the treatments followed flubendiamide 39.5 SC $(17.60 \%)$ and emamectin benzoate 5 SG $(18.14 \%)$, these treatments were on par with each other and there was no significant difference between these treatments. Highest fruit damage of 38.20 per cent was observed in untreated control. The insecticides in the decreasing order of their efficacy were chlorantraniliprole 18.5 SC > flubendiamide 39.5 $\mathrm{SC}>$ emamectin benzoate $5 \mathrm{SG}>$ cyantraniliprole $10.26 \mathrm{OD}>$ bifenthrin $10 \mathrm{EC}$ $>$ spinosad $45 \mathrm{SC}$ (Table 3 ).

Among the different treatments, emamectin benzoate $5 \mathrm{SG}$ recorded significantly highest fruit yield (25.90 t/ha) compared to rest of the treatments. The next best treatments were flubendiamide $39.5 \mathrm{SC} \quad(23.7 \mathrm{t} / \mathrm{ha})$ and chlorantraniliprole 18.5 SC (21.8 t/ha) which were on par with each other. The lowest yield of $10.40 \mathrm{t} /$ ha was recorded in untreated check (Table 4).

The cost economics revealed that emamectin benzoate $5 \mathrm{SG}$ registered higher net profit of Rs. $141884 \mathrm{ha}^{-1}$ with B: C ratio (3.17). This was followed by flubendiamide $39.5 \mathrm{SC}$, chlorantraniliprole 18.5 SC, cyantraniliprole 10.26 OD, bifenthrin $10 \mathrm{EC}$ and spinosad 45 SC registered net profit of Rs. 125604, 108179.84, 92325.67, 79256 and 71937.58 $\mathrm{ha}^{-1}$ with $\mathrm{B}$ : C ratio of 2.96, 2.63, 2.48, 2.28 and 2.12 , respectively and the lowest net profit of Rs. 15084 with B: C ratio (1.25) was recorded in untreated control (Table 5). 
Table.1 Efficacy of new insecticides against brinjal shoot and fruit borer, L. orbonalis during Kharif 2017

\begin{tabular}{|c|c|c|c|c|c|c|c|c|}
\hline \multirow{3}{*}{$\begin{array}{l}\text { Sl. } \\
\text { No. }\end{array}$} & \multirow[t]{3}{*}{ Treatments } & \multirow{3}{*}{$\begin{array}{c}\text { g a.i / } \\
\text { ha }\end{array}$} & \multicolumn{4}{|c|}{$\%$ Shoot damage } & \multirow[t]{3}{*}{ Mean } & \multirow{3}{*}{$\begin{array}{c}\text { Per cent reduction over } \\
\text { control }\end{array}$} \\
\hline & & & \multicolumn{4}{|c|}{ First spray } & & \\
\hline & & & $1 \mathrm{DBS}$ & 3 DAS & 7 DAS & 14 DAS & & \\
\hline 1 & Emamectin benzoate $5 \mathrm{SG}$ & 200 & $\frac{7.79}{(16.21)}$ & $\frac{6.33}{(14.57)^{\mathrm{a}}}$ & $\frac{3.31}{(10.48)^{\mathrm{a}}}$ & $\frac{6.60}{(14.89)^{\mathrm{a}}}$ & 5.41 & 63.10 \\
\hline 2 & Flubendiamide $39.5 \mathrm{SC}$ & 75 & $\begin{array}{c}7.70 \\
(16.11)\end{array}$ & $\begin{array}{c}6.77 \\
(15.08)^{\mathrm{ab}}\end{array}$ & $\begin{array}{c}3.99 \\
(11.52)^{\mathrm{ab}}\end{array}$ & $\begin{array}{c}7.12 \\
(15.48)^{\mathrm{ab}}\end{array}$ & 5.96 & 59.35 \\
\hline 3 & Chlorantraniliprole $18.5 \mathrm{SC}$ & 62.5 & $\begin{array}{c}8.46 \\
(16.91)\end{array}$ & $\begin{array}{c}7.00 \\
(15.34)^{\mathrm{abc}}\end{array}$ & $\begin{array}{c}3.59 \\
(10.92)^{\mathrm{ab}}\end{array}$ & $\begin{array}{c}6.91 \\
(15.24)^{\mathrm{ab}}\end{array}$ & 5.83 & 60.23 \\
\hline 4 & Cyantraniliprole 10 OD & 500 & $\begin{array}{c}9.27 \\
(17.73)\end{array}$ & $\begin{array}{c}8.21 \\
(16.65)^{\mathrm{bcd}}\end{array}$ & $\begin{array}{c}5.56 \\
(13.64)^{c}\end{array}$ & $\begin{array}{c}8.37 \\
(16.82)^{\mathrm{c}}\end{array}$ & 7.38 & 49.66 \\
\hline 5 & Spinosad 45 SC & 62.5 & $\begin{array}{c}9.32 \\
(17.78) \\
\end{array}$ & $\begin{array}{c}9.80 \\
(18.24)^{\mathrm{de}}\end{array}$ & $\begin{array}{c}6.70 \\
(15.00)^{\mathrm{cd}}\end{array}$ & $\begin{array}{c}9.50 \\
(17.95)^{\mathrm{cd}}\end{array}$ & 8.67 & 40.86 \\
\hline 6 & Bifenthrin $10 \mathrm{EC}$ & 500 & $\begin{array}{c}8.20 \\
(16.64)\end{array}$ & $\begin{array}{c}8.44 \\
(16.89)^{\mathrm{cde}}\end{array}$ & $\begin{array}{c}6.34 \\
(14.58)^{\mathrm{cd}}\end{array}$ & $\begin{array}{c}8.8 \\
(17.26)^{\mathrm{cd}}\end{array}$ & 7.86 & 46.38 \\
\hline \multirow[t]{3}{*}{7} & Untreated control & -- & $\begin{array}{c}9.47 \\
(17.47)\end{array}$ & $\begin{array}{c}12.04 \\
(20.30)^{\mathrm{f}}\end{array}$ & $\begin{array}{c}15.54 \\
(23.22)^{\mathrm{e}}\end{array}$ & $\begin{array}{c}16.4 \\
(23.89)^{\mathrm{e}}\end{array}$ & 14.66 & -- \\
\hline & $\operatorname{S.Em}( \pm)$ & -- & \multirow[t]{2}{*}{ NS } & 0.56 & $\mathbf{0 . 5 3}$ & 0.42 & 0.50 & -- \\
\hline & CD@5\% & -- & & 1.73 & 1.62 & 1.28 & 1.54 & -- \\
\hline
\end{tabular}

Figures in parentheses are arc sine transformed values 
Table.2 Efficacy of new insecticides against brinjal shoot and fruit borer, L. orbonalis during Kharif 2017

\begin{tabular}{|c|c|c|c|c|c|c|c|c|}
\hline \multirow{3}{*}{$\begin{array}{l}\text { Sl. } \\
\text { No. }\end{array}$} & \multirow[t]{3}{*}{ Treatments } & \multirow[t]{3}{*}{ g a.i / ha } & \multicolumn{4}{|c|}{ \% Shoot damage } & \multirow[t]{3}{*}{ Mean } & \multirow{3}{*}{$\begin{array}{l}\text { Per cent } \\
\text { reduction } \\
\text { over } \\
\text { control }\end{array}$} \\
\hline & & & \multicolumn{4}{|c|}{ Second spray } & & \\
\hline & & & $1 \mathrm{DBS}$ & 3 DAS & 7 DAS & 14 DAS & & \\
\hline 1 & Emamectin benzoate $5 \mathrm{SG}$ & 200 & $\begin{array}{c}7.72 \\
(16.13)\end{array}$ & $\begin{array}{c}7.07 \\
(15.42)^{\mathrm{ab}}\end{array}$ & $\begin{array}{c}3.33 \\
(10.51)^{\mathrm{a}}\end{array}$ & $\begin{array}{c}8.57 \\
(17.03)^{\mathrm{a}}\end{array}$ & 6.32 & 64.65 \\
\hline 2 & Flubendiamide $39.5 \mathrm{SC}$ & 75 & $\begin{array}{c}7.60 \\
(16.00)\end{array}$ & $\begin{array}{c}7.16 \\
(15.22)^{\mathrm{a}}\end{array}$ & $\begin{array}{c}4.28 \\
(11.93)^{b}\end{array}$ & $\begin{array}{c}9.39 \\
(17.85)^{\mathrm{ab}}\end{array}$ & 6.94 & 61.19 \\
\hline 3 & Chlorantraniliprole $18.5 \mathrm{SC}$ & 62.5 & $\begin{array}{c}8.37 \\
(16.82)\end{array}$ & $\begin{array}{c}7.26 \\
(15.63)^{\mathrm{abc}}\end{array}$ & $\begin{array}{c}4.59 \\
(12.38)^{b}\end{array}$ & $\begin{array}{c}9.71 \\
(18.16)^{b c}\end{array}$ & 7.19 & 59.79 \\
\hline 4 & Cyantraniliprole 10 OD & 500 & $\begin{array}{c}9.12 \\
(17.58)\end{array}$ & $\begin{array}{c}8.99 \\
(17.45)^{\mathrm{de}}\end{array}$ & $\begin{array}{c}7.65 \\
(16.06)^{c}\end{array}$ & $\begin{array}{c}10.57 \\
(18.97)^{\mathrm{c}}\end{array}$ & 9.07 & 49.27 \\
\hline 5 & Spinosad 45 SC & 62.5 & $\begin{array}{c}9.22 \\
(17.68)\end{array}$ & $\begin{array}{c}9.14 \\
(17.60)^{\mathrm{de}}\end{array}$ & $\begin{array}{c}7.99 \\
(16.42)^{\mathrm{cd}}\end{array}$ & $\begin{array}{c}12.03 \\
(20.29)^{d}\end{array}$ & 9.72 & 45.64 \\
\hline 6 & Bifenthrin $10 \mathrm{EC}$ & 500 & $\begin{array}{c}8.12 \\
(16.56)\end{array}$ & $\begin{array}{c}8.08 \\
(16.51)^{\mathrm{abcd}}\end{array}$ & $\begin{array}{c}7.79 \\
(16.21)^{\mathrm{cd}}\end{array}$ & $\begin{array}{c}11.76 \\
(20.06)^{d}\end{array}$ & 9.21 & 48.49 \\
\hline \multirow[t]{3}{*}{7} & Untreated control & -- & $\begin{array}{c}9.36 \\
(17.82)\end{array}$ & $\begin{array}{c}13.52 \\
(21.57)^{\mathrm{f}}\end{array}$ & $\begin{array}{c}17.33 \\
(24.60)^{\mathrm{e}}\end{array}$ & $\begin{array}{c}22.79 \\
(28.51)^{\mathrm{e}}\end{array}$ & 17.88 & -- \\
\hline & S. $\operatorname{Em}( \pm)$ & -- & \multirow[t]{2}{*}{ NS } & 0.44 & 0.30 & 0.32 & 0.35 & -- \\
\hline & CD@ $5 \%$ & -- & & 1.37 & 0.93 & 0.99 & 1.09 & -- \\
\hline
\end{tabular}

DBS - Day before spraying;

DAS - Days after spraying;

NS - Non significant

Figures in parentheses are arc sine transformed values 
Table.3 Efficacy of new insecticides against brinjal shoot and fruit borer, L. orbonalis during Kharif 2017

\begin{tabular}{|c|c|c|c|c|c|c|c|c|}
\hline \multirow[t]{3}{*}{ Sl. No. } & \multirow[t]{3}{*}{ Treatments } & \multirow[t]{3}{*}{ g a.i / ha } & \multicolumn{4}{|c|}{$\%$ fruit damage } & \multirow[t]{3}{*}{ Mean } & \multirow{3}{*}{$\begin{array}{c}\text { Per cent } \\
\text { reduction } \\
\text { over } \\
\text { control }\end{array}$} \\
\hline & & & \multicolumn{4}{|c|}{ Third spray } & & \\
\hline & & & 1 DBS & 3 DAS & 7 DAS & 14 DAS & & \\
\hline 1 & Emamectin benzoate $5 \mathrm{SG}$ & 200 & $\begin{array}{c}23.00 \\
(28.66)\end{array}$ & $\begin{array}{c}16.05 \\
(23.62)^{b}\end{array}$ & $\begin{array}{c}14.72 \\
(22.56)^{b}\end{array}$ & $\begin{array}{c}18.14 \\
(25.21)^{b}\end{array}$ & 16.30 & 54.16 \\
\hline 2 & Flubendiamide $39.5 \mathrm{SC}$ & 75 & $\begin{array}{c}25.20 \\
(30.13)\end{array}$ & $\begin{array}{c}15.36 \\
(23.08)^{\mathrm{ab}}\end{array}$ & $\begin{array}{c}14.37 \\
(22.28)^{b}\end{array}$ & $\begin{array}{c}17.60 \\
(24.80)^{b}\end{array}$ & 15.78 & 55.62 \\
\hline 3 & Chlorantraniliprole $18.5 \mathrm{SC}$ & 62.5 & $\begin{array}{c}24.62 \\
(29.75)\end{array}$ & $\begin{array}{c}14.28 \\
(22.20)^{\mathrm{a}}\end{array}$ & $\begin{array}{c}12.16 \\
(20.41)^{\mathrm{a}}\end{array}$ & $\begin{array}{c}16.52 \\
(23.98)^{\mathrm{a}}\end{array}$ & 14.32 & 59.73 \\
\hline 4 & Cyantraniliprole 10 OD & 500 & $\begin{array}{c}24.02 \\
(29.35)\end{array}$ & $\begin{array}{c}23.99 \\
(29.33)^{c}\end{array}$ & $\begin{array}{c}24.49 \\
(29.66)^{c}\end{array}$ & $\begin{array}{c}28.60 \\
(32.33)^{d}\end{array}$ & 25.69 & 27.76 \\
\hline 5 & Spinosad $45 \mathrm{SC}$ & 62.5 & $\begin{array}{c}26.14 \\
(30.75)\end{array}$ & $\begin{array}{c}25.96 \\
(30.63)^{\mathrm{e}}\end{array}$ & $\begin{array}{c}25.88 \\
(30.58)^{d}\end{array}$ & $\begin{array}{c}30.03 \\
(33.23)^{\mathrm{e}}\end{array}$ & 27.29 & 23.26 \\
\hline 6 & Bifenthrin $10 \mathrm{EC}$ & 500 & $\begin{array}{c}26.05 \\
(30.69)\end{array}$ & $\begin{array}{c}25.78 \\
(30.51)^{d}\end{array}$ & $\begin{array}{c}24.40 \\
(29.60)^{\mathrm{c}}\end{array}$ & $\begin{array}{c}27.50 \\
(31.63)^{\mathrm{c}}\end{array}$ & 25.89 & 27.19 \\
\hline \multirow[t]{3}{*}{7} & Untreated control & -- & $\begin{array}{c}25.22 \\
(30.15)\end{array}$ & $\begin{array}{c}33.18 \\
(35.17)^{\mathrm{f}}\end{array}$ & $\begin{array}{c}35.31 \\
(36.46)^{\mathrm{e}}\end{array}$ & $\begin{array}{c}38.20 \\
(38.17)^{\mathrm{f}}\end{array}$ & 35.56 & -- \\
\hline & S. $\operatorname{Em}( \pm)$ & -- & \multirow[t]{2}{*}{ NS } & 0.30 & 0.23 & 0.21 & 0.25 & -- \\
\hline & CD@ $5 \%$ & -- & & 0.92 & 0.70 & 0.66 & 0.76 & -- \\
\hline
\end{tabular}

Figures in parentheses are arc sine transformed values 
Table.4 Efficacy of new insecticides against brinjal shoot and fruit borer, L. orbonalis during Kharif 2017

\begin{tabular}{|c|c|c|c|}
\hline Treatments & Dose (g a.i / ha) & $\begin{array}{l}\text { Fruit yield } \\
\text { (t/ ha) }\end{array}$ & $\begin{array}{c}\text { Per cent increase over } \\
\text { control }\end{array}$ \\
\hline Emamectin benzoate 5 SG & 200 & 25.90 & 59.85 \\
\hline Flubendiamide $39.5 \mathrm{SC}$ & 75 & 23.70 & 56.12 \\
\hline Chlorantraniliprole $18.5 \mathrm{SC}$ & 62.5 & 21.80 & 52.30 \\
\hline Cyantraniliprole 10 OD & 500 & 19.30 & 46.11 \\
\hline Spinosad 45 SC & 62.5 & 17.00 & 38.82 \\
\hline Bifenthrin $10 \mathrm{EC}$ & 500 & 17.60 & 40.90 \\
\hline Untreated control & -- & 10.40 & -- \\
\hline S.Em $( \pm)$ & -- & 0.81 & -- \\
\hline CD@ $5 \%$ & -- & 2.49 & -- \\
\hline
\end{tabular}


Table.5 Cost economics for the management of brinjal shoot and fruit borer in brinjal during Kharif 2017

\begin{tabular}{|c|c|c|c|c|c|c|c|c|}
\hline Treatments & $\begin{array}{c}\text { Dose } \\
\text { g a.i/ ha }\end{array}$ & $\begin{array}{l}\text { Yield } \\
\text { (t/ ha) }\end{array}$ & $\begin{array}{c}\text { Plant } \\
\text { protection } \\
\text { cost }(\mathrm{Rs} / \\
\text { ha) }\end{array}$ & $\begin{array}{c}\text { Other } \\
\text { Expenditure } \\
\text { (Rs/ ha) }\end{array}$ & $\begin{array}{l}\text { Total cost } \\
\text { of } \\
\text { Production } \\
\text { (Rs/ ha) }\end{array}$ & $\begin{array}{c}\text { Gross } \\
\text { returns } \\
\text { (Rs/ ha) }\end{array}$ & $\begin{array}{l}\text { Net returns } \\
\quad(\mathrm{Rs} / \mathrm{ha})\end{array}$ & $\begin{array}{l}\text { B: } C \\
\text { ratio }\end{array}$ \\
\hline Emamectin benzoate 5 SG & 200 & 25.90 & 5200 & 60116 & 65316 & 207200 & 141884 & 3.17 \\
\hline Flubendiamide $39.5 \mathrm{SC}$ & 75 & 23.70 & 3880 & 60116 & 63996 & 189600 & 125604 & 2.96 \\
\hline Chlorantraniliprole 18.5 SC & 62.5 & 21.80 & 6104.16 & 60116 & 66220.16 & 174400 & 108179.84 & 2.63 \\
\hline Cyantraniliprole 10 OD & 500 & 19.30 & 1958.33 & 60116 & 62074.33 & 154400 & 92325.67 & 2.48 \\
\hline Spinosad 45 SC & 62.5 & 17.00 & 3946.42 & 60116 & 64062.42 & 136000 & 71937.58 & 2.12 \\
\hline Bifenthrin $10 \mathrm{EC}$ & 500 & 17.60 & 1428 & 60116 & 61544 & 140800 & 79256 & 2.28 \\
\hline Untreated control & -- & 10.40 & -- & 60116 & 60116 & 83200 & 23084 & 1.25 \\
\hline
\end{tabular}


Int.J.Curr.Microbiol.App.Sci (2019) 8(3): 2582-2592

Fig.1 Yield and B: C ratio of insecticides during Kharif 2017

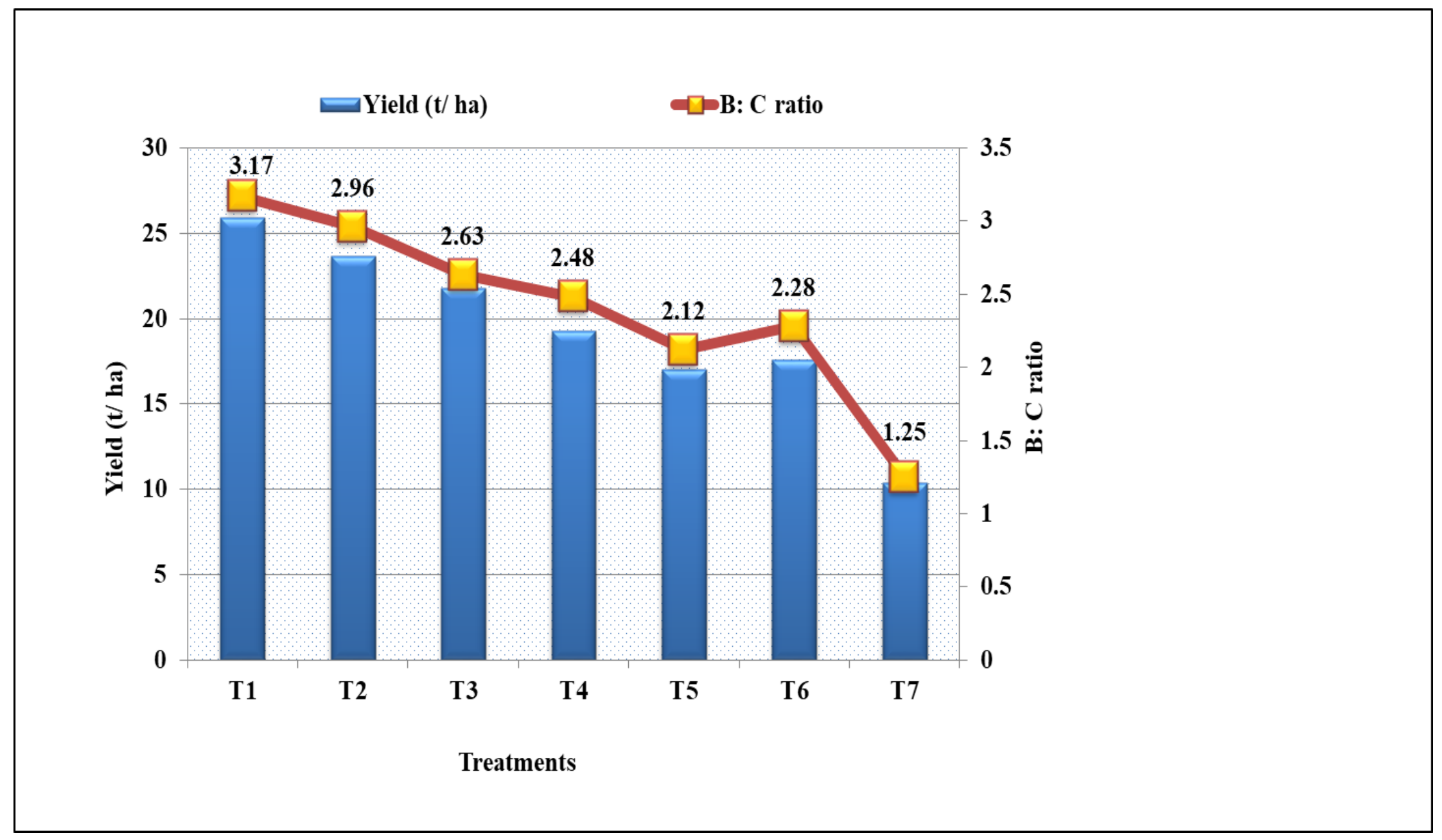


New novel insecticides were evaluated for their efficacy to manage the BSFB and compared with recommended insecticides. Control plots had significantly higher BSFB infestation at three, seven and fourteen days after spray as compared to that on insecticide treated plots. However, among tested insecticides emamectin benzoate, chlorantraniprole $18.5 \mathrm{SC}$ and flubendiamide were relatively more effective compared to remaining treatments first and second spray.

The new generation chemicals used in this study belong to different groups of IRAC (Insecticide resistance action committee) and offer unique modes of action. The findings of present studies indicated that Chlorantraniliprole $\quad 18.50 \quad$ SC and/or Flubendiamide $39.35 \mathrm{SC}$ proved better for the management of BSFB. These findings corroborate with the results obtained by Mishra (2008) and Jagginavar et al. (2009) where they opined that Chlorantraniliprole 18.50 SC and/or Flubendiamide 39.35 SC were superior in controlling BSFB.

Present results are also in accordance with the study conducted by Shah et al. (2012), who found that emamectin benzoate and flubendiamide were promising insecticides to lower brinjal shoot and fruit borer infestation and produce high fruit yield (Latif et al. 2009) also suggested the application of flubendiamide in combination with mechanical control, potash and field sanitation, for reducing fruit and shoot infestation. Our findings also confirm the results of the studies conducted by Latif et al. (2010), who found that flubendiamide caused maximum larval mortality of BSFB in laboratory trials while field trials reduced brinjal shoot and fruit infestation by $70-80$ per cent.

After third spray, control plots had significantly higher infestation as compared to that of insecticides applied plot. Among the insecticides treatments Chlorantraniliprole was the most effective having the least fruit infestation at three, seven and fourteen days after spray. Chlorantraniliprole was most effective insecticide against BSFB at three, seven and fourteen days after spray followed by flubendiamide and emamectin benzoate.

The results of the present study support the findings of several previous studies. Anil and Sharma (2010), Sharma and Sharma (2010), Chatterjee and Mondal (2012) and Shah et al. (2012) who reported that emamectin benzoate was the most effective insecticide in reducing BSFB infestation and increasing marketable fruit yield.

Among the different treatments, emamectin benzoate 5 SG recorded significantly highest fruit yield (25.90 t/ha) compared to rest of the treatments. The next best treatments were flubendiamide $39.5 \%$ SC (23.7 t/ha) and chlorantraniliprole 18.5 SC (21.8 t/ha) which were on par with each other. Whereas, in treatments cyantraniliprole 10 OD (19.30 t/ha), bifenthrin $10 \%$ EC (17.6 t/ha) and spinosad 45 SC (17 t/ha) were the next best treatments in recording fruit yield (Fig. 1).

The lowest yield of $10.40 \mathrm{t} / \mathrm{ha}$ was recorded in untreated check. The results of the present study support the findings of several previous study Shirale et al. (2012) who reported that the total fruit yield was significantly higher in the plots sprayed with Chlorantraniliprole 18.50 SC (528.52 q/ ha).

The results on the cost economics of various treatments revealed that emamectin benzoate 5 $\%$ SG and flubendiamide 39.5 SC registered a high B: C ratio of 3.17 and 2.96, respectively (Fig. 1). The results of present investigation were almost in line with findings of Biradar et al. (2001) they noticed that the B: $\mathrm{C}$ ratio for flubendiamide $39.5 \mathrm{SC}$ was 4.44 .

Over all, it can be concluded that emamectin benzoate $5 \mathrm{SG}$, flubendiamide $39.5 \mathrm{SC}$ and chlorantraniliprole $\quad 18.5 \quad \mathrm{SC}$ recorded comparatively lower shoot and fruit damage and higher fruit yield and were found promising insecticides for the management of BSFB. 


\section{References}

Anil and Sharma, P. C., 2010, Bioefficacy of insecticides against Leucinodes orbonalis (Guenee) on brinjal. J. environ. Biol., 31: 399- 402.

Anonymous, 2016. National Horticultural Mission- 2016.

Biradar, A. P., Teggelli, R. G., Patil, D. R. and Balikai, R. A., 2001, Evaluation of cypermethrin $3 \mathrm{EC}+$ quinalphos $20 \mathrm{EC}$ against brinjal shoot and fruit borer. Karnataka J. Agri. Sci., 14: 369- 372.

Chatterjee, M. L. and Mondal, S., 2012, Sustainable management of key lepidopteran insect pests of vegetables. ISHS Acta Horticult.

Hemi, M. A., 1955, Effect of borer attack on the vitamin C content of brinjal. Pak. J. Health, 4: 223-224.

Jagginavar, S. B., Sunitha, N. D. and Biradar, A. P., 2009, Bioefficacy of Flubendiamide 48 SC against brinjal fruit and shoot borer, Leucinodes orbonalis Guen. Karnataka J. agric. Sci., 22: 712713.

Latif, M. A., Rahman, M. M., Alam, M. Z. and Hossain, M. M., 2009, Evaluation of Flubenbendiamide as an IPM component for the management of brinjal shoot and fruit borer, Leucinodes orbonalis Guenee. Mun. Ent. Zool., 4: 257-267.

Latif, M. A., Rahman, M. M., Alam, M. Z. and Hossain, M. M., 2010, Evaluation of Flubenbendiamide as an IPM component for the management of brinjal shoot and fruit borer, Leucinodes orbonalis Guenee. Mun. Ent. Zool., 5: 352-368.

Mishra, H. P., 2008, New promising insecticides for the management of brinjal shoot and fruit borer, Leucinodes orbonalis (Guenee). Pest Manage. Hort. Ecosys., 14: 140- 147.

Raghupathy, A., Palanisamy, S., Chandramohan, N. and Gunathilagaraj, K., 1997, A guide on crop pests. Sooriya desk Top Publishers, Coimbatore, pp. 264.

Reddy, E. S. G. and Srinivasa, 2004, Management of shoot and fruit borer, Leucinodes orbonalis (Guen.) in brinjal using botanicals or oils. Pestology, 28: 50- 52.

Shah, K. D., Bharpoda, T. M. and Jhala, R. C., 2012, Bio-efficacy of newer molecules of insecticides against brinjal shoot and fruit borer, Leucinodes orbonalis (Guen). An inter. e-J., 1(2): 186-200.

Sharma, A. and Sharma, P. C., 2010, Bioefficacy of insecticides against Leucinodes orbonalis on brinjal. $J$. environ. Biol., 31: 399-402.

Singh,Y. P., Gangwar, S. K. and Thakur, N. S. A., 1962, Persistence of endosulfan in/on maize at medium high altitude hills of Meghalaya. Indian J. Plant Prot., 20(2): 195-197.

Subbaratnam, G. V. and Butani, D. K., 1982, Chemical control of insect pest complex of brinjal. Entomologica, 7: 97-100.

Vevai, G. H., Khokhar, K. M. and Ashraf, M., 1970, Management of insect pests of Brinjal. Pak. J. Agric. Res., 23: 33-39.

Shirale, D., Patil, M., Zehr, U. and Parimi, S., 2012, Evaluation of newer insecticides for the management of brinjal fruit and shoot borer Leucinodes orbonalis (Guenee). Indian J. of Plant Prot., 40(4): 273-275

\section{How to cite this article:}

Shridhara, M., S.G. Hanchinal, A.G. Sreenivas, A.C. Hosamani and Nidagundi, J.M. 2019. Evaluation of Newer Insecticides for the Management of Brinjal Shoot and Fruit Borer Leucinodes orbonalis (Guenee) (Lepidoptera: Crambidae). Int.J.Curr.Microbiol.App.Sci. 8(03): 2582-2592. doi: https://doi.org/10.20546/ijcmas.2019.803.306 